\title{
Blowup of Jang's Equation at Outermost Marginally Trapped Surfaces
}

\author{
Jan Metzger ${ }^{\star}$ \\ Albert-Einstein-Institut, Am Mühlenberg 1, D-14476 Potsdam, Germany. \\ E-mail: jan.metzger@aei.mpg.de
}

Received: 30 January 2009 / Accepted: 24 July 2009

Published online: 8 October 2009 - (C) The Author(s) 2009. This article is published with open access at Springerlink.com

\begin{abstract}
The aim of this paper is to accurately describe the blowup of Jang's equation. First, we discuss how to construct solutions that blow up at an outermost MOTS. Second, we exclude the possibility that there are extra blowup surfaces in data sets with non-positive mean curvature. Then we investigate the rate of convergence of the blowup to a cylinder near a strictly stable MOTS and show exponential convergence with an identifiable rate near a strictly stable MOTS.
\end{abstract}

\section{Introduction}

This paper is concerned with the examination of the relation of Jang's equation to marginally outer trapped surfaces (MOTS). To set the perspective, we consider Cauchy data $(M, g, K)$ for the Einstein equations. Such data sets are 3-manifolds $M$ equipped with a Riemannian metric together with a symmetric bilinear form $K$ representing the second fundamental form of the time slice $M$ in space-time. A marginally outer trapped surface is a surface with $\theta^{+}=H+P=0$, where $H$ is the mean curvature of $\Sigma$ in $M$ and $P=\operatorname{tr} K-K(v, v)$ for the normal $v$ to $\Sigma$.

In the paper [AM07], inspired by an idea of Schoen [Sch04], we constructed MOTS in the presence of barrier surfaces by inducing a blow-up of Jang's equation. In this context, Jang's equation [SY81,Jan78] is an equation of prescribed mean curvature for the graph of a function in $M \times \mathbf{R}$. For details we refer to Sect. 2 .

In this note, we take a slightly different perspective. Consider a data set $(M, g, K)$ with a non-empty outer boundary $\partial^{+} M$ and assume that we are given the outermost MOTS $\Sigma$ in $(M, g, K)$. Here, outermost means that there is no other MOTS on the outside of $\Sigma$. From [AM07] it follows that $(M, g, K)$ always contains a unique such surface, or does not contain outer trapped surfaces at all, under the assumption that $\partial M$ is outer untrapped. As stated in Theorem 3.1, we show that there exists a solution $f$

* Research on this project started while the author was supported in part by a Feodor-Lynen Fellowship of the Humboldt Foundation. 
to Jang's equation that actually blows up at $\Sigma$, assuming that $\partial M$ is inner and outer untrapped. By blow-up we mean that outside from $\Sigma$ the function $f$ is such that graph $f$ is a smooth submanifold of $M \times \mathbf{R}$ with a cylindrical end converging to $\Sigma \times \mathbf{R}$. There is however a catch, as $f$ may blow up at other surfaces, too. These surfaces are marginally inner trapped. In Theorem 3.4 we show that the other blow-up surfaces can not occur if the data set has non-positive mean curvature.

To put the result in perspective note that if the dominant energy condition holds, the graph of $f$ is of non-negative Yamabe type and thus can be equipped with a (singular) metric of zero scalar curvature. This was used by Schoen and Yau in [SY81] to prove the positive mass theorem. Later Bray [Bra01] proposed to use Jang's equation to relate the Penrose conjecture in its general setting to the Riemannian Penrose inequality [HI01, Bra01] on a manifold constructed from Jang's graph. One of the main questions in this program is whether or not Jang's equation can be made to blow up at a specific MOTS. This question was raised in the literature, cf. for example [MÓM04] where this is discussed in the rotationally symmetric case. Here we give the positive answer that blow-up solutions exist at outermost MOTS. The author recently learned that the existence of the blow-up solution is used in [Khu09] to prove a Penrose-like inequality.

With the blow-up constructed, we can turn to the asymptotic behavior of the blowup itself. It has been shown in [SY81] that such a blow-up must be asymptotic to a cylinder over the outermost MOTS. In Theorems 4.2 and 4.4 we show that under the assumption of strict stability the convergence rate is exponential with a power directly related to the principal eigenvalue of the MOTS. The general idea is to show the existence of a super-solution with at most logarithmic blow-up of the desired rate. Turning the picture sideways yields exponential decay, when writing the solution as a graph over the cylinder in question. Furthermore, we show that beyond a certain decay rate, the solution must be trivial, thus exhibiting the actual rate.

We expect that the knowledge of these asymptotics is tied to the question whether the blow-up solution is unique. Furthermore note that the constant in the Penrose-like inequality in [Khu09] depends on the geometry of the solution. We thus expect that the value on this constant is related to the asymptotic behavior near the blow-up cylinder.

Before turning to these results, we introduce some notation in Sect. 2. Section 3 proceeds with the construction of the the blow-up. We will not go into details here, but emphasize the general idea and point to the results needed from the paper [AM07]. In Sect. 4 , we perform the calculation of the asymptotics.

\section{Preliminaries}

Let $(M, g, K)$ be an initial data set for the Einstein equations. That is $M$ is a 3-dimensional manifold, $g$ a Riemannian metric on $M$ and $K$ a symmetric 2-tensor. We do not require any energy condition to hold.

Assume that $\partial M$ is the disjoint union $\partial M=\partial^{-} M \cup \partial^{+} M$, where $\partial^{ \pm} M$ are smooth surfaces without boundary. We refer to $\partial^{-} M$ as the inner boundary and endow it with the normal vector $v$ pointing into $M$. The outer boundary $\partial^{+} M$ is endowed with the normal $v$ pointing out of $M$. We denote by $H[\partial M]$ the mean curvature of $\partial M$ with respect to the normal vector field $\nu$, and by $P[\partial M]=\operatorname{tr}_{\partial M} K$ the trace of the tensor $K$ restricted to the 2-dimensional surface $\partial M$. Then the inward and outward expansions of $\partial M$ are defined by

$$
\theta^{ \pm}[\partial M]=P[\partial M] \pm H[\partial M] .
$$

Assume that $\theta^{+}\left[\partial^{-} M\right]=0$, and that $\theta^{+}\left[\partial^{+} M\right]>0$ and $\theta^{-}\left[\partial^{+} M\right]<0$. 
If $\Sigma \subset M$ is a smooth, embedded surface homologous to $\partial^{+} M$, then $\Sigma$ bounds a region $\Omega$ together with $\partial^{+} M$. In this case, we define $\theta^{ \pm}[\Sigma]$ as above, where $H$ is computed with respect to the normal vector field pointing into $\Omega$ (that is in direction of $\partial^{+} M$ ). $\Sigma$ is called a marginally outer trapped surface (MOTS), if $\theta^{+}[\Sigma]=0$. We say that $\partial M$ is an outermost MOTS, if there is no other MOTS in $M$, which is homologous to $\partial^{+} M$. In [AM07] it is proved that for any initial data set $(M, g, K)$ which contains a MOTS, there is also an outermost MOTS surrounding it.

Let $\Sigma \subset M$ be a MOTS and consider a normal variation of $\Sigma$ in $M$, that is a map $F: \Sigma \times(-\varepsilon, \varepsilon) \rightarrow M$ such that $F(\cdot, 0)=\operatorname{id}_{\Sigma}$ and $\left.\frac{\partial}{\partial s}\right|_{s=0} F(p, s)=f v$, where $f$ is a function on $\Sigma$ and $v$ is the normal of $\Sigma$. Then the change of $\theta^{+}$is given by

$$
\left.\frac{\partial \theta^{+}[F(\Sigma, s)]}{\partial d s}\right|_{s=0}=L_{M} f,
$$

where $L_{M}$ is a quasi-linear elliptic operator of second order along $\Sigma$. It is given by

$$
L_{M} f=-\Delta f+2 S(\nabla f)+f\left(\operatorname{div} S-\left|\chi^{+}\right|^{2}-|S|^{2}+\frac{1}{2}^{\Sigma} \mathrm{Sc}-\mu-J(\nu)\right) .
$$

In this expression $\nabla$, div and $\Delta$ denote the gradient, divergence and Laplace-Beltrami operator tangential to $\Sigma$. The tangential 1-form $S$ is given by $S=K(\cdot, v)^{T}, \chi^{+}$is the bilinear form $\chi^{+}=A+K^{\Sigma}$, where $A$ is the second fundamental form of $\Sigma$ in $M$ and $K^{\Sigma}$ is the projection of $K$ to $T \Sigma \times T \Sigma$. Furthermore, ${ }^{\Sigma} \mathrm{Sc}$ denotes the scalar curvature of $\Sigma, \mu=\frac{1}{2}\left({ }^{M} \mathrm{Sc}-|K|^{2}+(\operatorname{tr} K)^{2}\right)$, and $J={ }^{M} \operatorname{div} K-d \operatorname{tr} K$. For a more detailed investigation of this operator we refer to [AMS05] and [AMS07].

The facts we will need here are that $L_{M}$ has a principal eigenvalue $\lambda$, which is real and has a one-dimensional eigenspace which is spanned by a positive function. If $\lambda$ is non-negative $\Sigma$ is called stable, and if $\lambda$ is positive, $\Sigma$ is called strictly stable. In particular, if $\Sigma$ is strictly stable as a MOTS, there exists an outward deformation strictly increasing $\theta^{+}$.

In $\bar{M}=M \times \mathbf{R}$, we consider Jang's equation [Jan78,SY81] for the graph of a function $f: M \rightarrow \mathbf{R}$. Let $N:=$ graph $f=\{(x, z): z=f(x)\}$. The mean curvature $\mathcal{H}[f]$ of $N$ with respect to the downward normal is given by

$$
\mathcal{H}[f]=\operatorname{div}\left(\frac{\nabla f}{\sqrt{1+|\nabla f|^{2}}}\right) .
$$

Define $\bar{K}$ on $\bar{M}$ by $\bar{K}_{(x, z)}(X, Y)=K_{x}(\pi X, \pi Y)$, where $\pi: T \bar{M} \rightarrow T M$ denotes the orthogonal projection onto the horizontal tangent vectors. Let

$$
\mathcal{P}[f]=\operatorname{tr}_{N} \bar{K}
$$

Then Jang's equation becomes

$$
\mathcal{J}[f]=\mathcal{H}[f]-\mathcal{P}[f]=0 .
$$




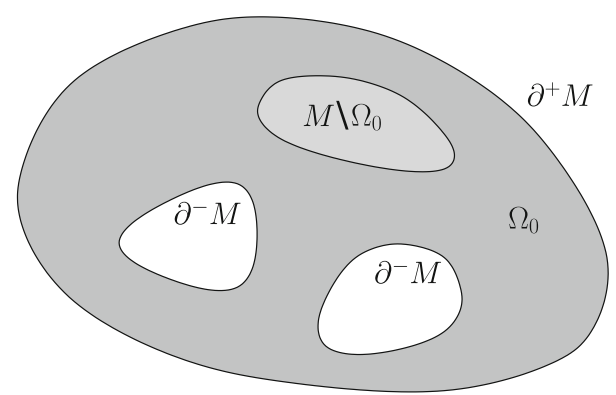

Fig. 1. The situation in Theorem 3.1. All of the shaded region belongs to $M$, whereas $f$ is only defined in $\Omega_{0}$

\section{The Blowup}

The main result of this section is that we can construct a solution to Jang's equation which blows up at the outermost MOTS in $(M, g, K)$ and has zero Dirichlet boundary data at $\partial^{+} M$. In fact, we chose the assumptions on the outer boundary $\partial^{+} M$ so that we can prescribe more general Dirichlet data there. The focus here lies on the blow-up in the interior, so that we do not investigate the optimal conditions for $\partial^{+} M$.

Theorem 3.1. If $(M, g, K)$ is an initial data set with $\partial M=\partial^{-} M \cup \partial^{+} M$ such that $\partial^{-} M$ is an outermost MOTS, $\theta^{+}\left[\partial^{+} M\right]>0$ and $\theta^{-}\left[\partial^{+} M\right]<0$, then there exists an open set $\Omega_{0} \subset M$ and a function $f: \Omega_{0} \rightarrow \mathbf{R}$ such that

1. $\overline{M \backslash \Omega_{0}}$ does not intersect $\partial M$,

2. $\theta^{-}\left[\partial \Omega_{0}\right]=0$ with respect to the normal vector pointing into $\Omega_{0}$,

3. $\mathcal{J}[f]=0$,

4. $N^{+}=$graph $f \cap M \times \mathbf{R}^{+}$is asymptotic to the cylinder $\partial^{-} M \times \mathbf{R}^{+}$,

5. $N^{-}=\operatorname{graph} f \cap M \times \mathbf{R}^{-}$is asymptotic to the cylinder $\partial \Omega_{0} \times \mathbf{R}^{-}$, and

6. $\left.f\right|_{\partial^{+} M}=0$.

For data sets $(M, g, K)$ which do not contain surfaces with $\theta^{-}=0$, the above theorem implies the following result.

Corollary 3.2. If $(M, g, K)$ is as in Theorem 3.1, and in addition there are no subsets $\Omega \subset M$ with $\theta^{-}[\partial \Omega]=0$ with respect to the normal pointing out of $\Omega$, then there exists a function $f: M \rightarrow \mathbf{R}$ such that

1. $\mathcal{J}[f]=0$,

2. $N=\operatorname{graph} f$ is asymptotic to the cylinder $\partial^{-} M \times \mathbf{R}^{+}$,

3. $\left.f\right|_{\partial^{+} M}=0$.

Remark 3.3. Analogous results hold if $(M, g, K)$ is asymptotically flat with appropriate decay of $g$ and $K$ instead of having an outer boundary $\partial^{+} M$. Then the assertion $f \mid{ }_{\partial^{+} M}=0$ in Theorem 3.1 has to be replaced by $f(x) \rightarrow 0$ as $x \rightarrow \infty$.

The proof of Theorem 3.1 is largely based on the tools developed in [SY81 and AM07]. Thus we will not include all details here, but provide a summary, which facts will have to be used. 
Proof. We will assume that $(M, g, K)$ is embedded into $\left(M^{\prime}, g^{\prime}, K^{\prime}\right)$ which extends $M$ beyond the boundary $\partial^{-} M$ such that $\partial^{-} M$ lies in the interior of $M^{\prime}$, without further requirements.

Let $\partial^{-} M=\cup_{i=1}^{N} \Sigma_{i}$ where the $\Sigma_{i}$ are the connected components of $\partial M$. As $\partial M$ is an outermost MOTS, each of the $\Sigma_{i}$ is stable [AM07, Cor. 5.3].

Following the proof of [AM07, Th. 5.1], we deform $\partial^{-} M$ to a surface $\Sigma_{s}$ by pushing the components $\Sigma_{i}$ out of $M$, into the extension $M^{\prime}$. To this end, let $\phi_{i}>0$ be the principal eigenfunction of the stability operator of $\Sigma_{i}$ and extend the vector field $X_{i}=-\phi_{i} v_{i}$ to a neighborhood of $\Sigma_{i}$ in $M^{\prime}$. Flowing $\Sigma_{i}$ by $X_{i}$ yields a family of surfaces $\Sigma_{i}^{s}, s \in[0, \varepsilon)$ so that the $\Sigma_{i}^{s}$ form a smooth foliation for small enough $\varepsilon$ with $\Sigma_{i}^{s} \in M^{\prime} \backslash M$. If $\Sigma_{i}$ is strictly stable then

$$
\left.\frac{\partial}{\partial s}\right|_{s=0} \theta^{+}\left[\Sigma_{s}\right]=-\lambda \phi<0,
$$

where $\lambda$ is the principal eigenvalue of $\Sigma_{i}$. Thus, for small enough $\varepsilon$, we have $\theta^{+}\left[\Sigma_{i}^{s}\right]<0$ for all $s \in(0, \varepsilon)$.

If $\Sigma_{i}$ has principal eigenvalue $\lambda=0$, then the $\Sigma_{i}^{s}$ satisfy

$$
\left.\frac{\partial}{\partial s}\right|_{s=0} \theta^{+}\left[\Sigma_{s}\right]=0 .
$$

In this case it is possible to change the data $K^{\prime}$ on $\Sigma_{i}^{s}$ as follows:

$$
\tilde{K}=K^{\prime}-\frac{1}{2} \psi(s) \gamma_{s},
$$

where $\gamma_{s}$ is the metric on $\Sigma_{s}$ and $\psi$ is a smooth function $\psi:[0, \varepsilon] \rightarrow \mathbf{R}$. The operator $\tilde{\theta}^{+}$, which means $\theta^{+}$computed with respect to the data $\tilde{K}$ instead of $K^{\prime}$, satisfies

$$
\tilde{\theta}^{+}\left[\Sigma_{i}^{S}\right]=\theta^{+}\left[\Sigma_{i}^{S}\right]-\psi(s) .
$$

It is clear from Eq. (3.1) that $\psi$ can be chosen such that $\psi(0)=\psi^{\prime}(0)=0$ and $\tilde{\theta}^{+}\left[\Sigma_{i}^{s}\right]<0$ for all $s \in(0, \varepsilon)$ provided $\varepsilon$ is small enough. Then $\tilde{K}$ is $C^{1,1}$ when extended by $K$ to the rest of $M$.

Replace each original boundary component $\Sigma_{i}$ of $M$ by a surface $\Sigma_{i}^{\varepsilon}$ as constructed above, and replace $K^{\prime}$ with $\tilde{K}$, such that the following properties are satisfied. Let $\tilde{M}$ denote the manifold with boundary components $\Sigma_{i}^{\varepsilon}$ resulting from this procedure. Thus we construct from $(M, g, K)$ a data set $\left(\tilde{M}, g^{\prime}, \tilde{K}\right)$ with the following properties:

1. $M \subset \tilde{M}$ with $\left.g^{\prime}\right|_{M}=g,\left.\tilde{K}\right|_{M}=K$, and $\partial^{+} M=\partial^{+} \tilde{M}$,

2. $\theta^{+}\left[\partial^{-} \tilde{M}\right]<0$, and

3. the region $\tilde{M} \backslash M$ is foliated by surfaces $\Sigma_{s}$ with $\theta^{+}\left(\Sigma_{s}\right)<0$.

The method developed in Sect. 3.2 in [AM07] now allows the modification of the data $\left(\tilde{M}, g^{\prime}, \tilde{K}\right)$ to a new data set, which we also denote by $(\tilde{M}, \tilde{g}, \tilde{K})$, although $\tilde{K}$ changes in this step. This data set has the following properties:

1. $M \subset \tilde{M}$ with $\left.g^{\prime}\right|_{M}=g,\left.\tilde{K}\right|_{M}=K$, and $\partial^{+} M=\partial^{+} \tilde{M}$,

2. $\theta^{+}\left[\partial^{-} \tilde{M}\right]<0$,

3. $H\left[\partial^{-} \tilde{M}\right]>0$, where $H$ is the mean curvature of $\partial^{-} M$ with respect to the normal pointing out of $\partial^{-} \tilde{M}$,

4. the region $\tilde{M} \backslash M$ is foliated by surfaces $\Sigma_{s}$ with $\theta^{+}\left(\Sigma_{S}\right)<0$. 
By Sect. 3.3 in [AM07] this enables us to solve the boundary value problem

$$
\begin{cases}\mathcal{J}\left[f_{\tau}\right]=\tau f_{\tau} & \text { in } \tilde{M} \\ f_{\tau}=\frac{\delta}{2 \tau} & \text { on } \partial^{-} \tilde{M} \\ f_{\tau}=0 & \text { on } \partial^{+} \tilde{M}\end{cases}
$$

where $\delta$ is a lower bound for $H$ on $\partial^{-} M$. The solvability of this equation follows, provided an estimate for the gradient at the boundary can be found. The barrier construction at $\partial^{-} \tilde{M}$ was carried out in detail in [AM07], whereas the barrier construction at $\partial^{+} \tilde{M}$ is standard due to the stronger requirement that $\theta^{+}\left[\partial^{+} M\right]>0$ and $\theta^{-}\left[\partial^{+} M\right]<0$.

The solution $f_{\tau}$ to Eq. (3.2) satisfies an estimate of the form

$$
\sup _{\tilde{M}}\left|f_{\tau}\right|+\sup _{\tilde{M}}\left|\nabla f_{\tau}\right| \leq \frac{C}{\tau},
$$

where $C$ is a constant depending only on the data $(\tilde{M}, \tilde{g}, \tilde{K})$ but not on $\tau$.

The gradient estimate implies in particular that there exists an $\varepsilon>0$ independent of $\tau$ such that

$$
f_{\tau}(x) \geq \frac{\delta}{4 \tau} \quad \forall x \text { with } \operatorname{dist}\left(x, \partial^{-} \tilde{M}\right)
$$

The graphs $N_{\tau}$ have uniformly bounded curvature in $\tilde{M} \times \mathbf{R}$ away from the boundary. This allows the extraction of a sequence $\tau_{i} \rightarrow 0$ such that the $N_{\tau_{i}}$ converge to a manifold $N$, cf. [AM07, Prop. 3.8], [SY81, Sect. 4]. This convergence determines three open subsets of $\tilde{M}$ :

$$
\begin{aligned}
\Omega_{-} & :=\left\{x \in M: f_{\tau_{i}}(x) \rightarrow-\infty \text { locally uniformly as } i \rightarrow \infty\right\}, \\
\Omega_{0} & :=\left\{x \in M: \limsup _{i \rightarrow \infty}\left|f_{\tau_{i}}(x)\right|<\infty\right\} \\
\Omega_{+} & :=\left\{x \in M: f_{\tau_{i}}(x) \rightarrow \infty \text { locally uniformly as } i \rightarrow \infty\right\} .
\end{aligned}
$$

From the fact that the $f_{\tau}$ blow up near $\partial^{-} \tilde{M}$, we have that $\Omega_{+} \neq \emptyset$ and $\Omega_{+}$contains a neighborhood of $\partial^{-} \tilde{\Omega}$. As already noted in [SY81] $\partial \Omega_{+} \backslash \partial \tilde{M}$ consists of MOTS. As the region $\tilde{M} \backslash M$ is foliated by surfaces with $\theta^{+}<0$, we must have that $\Omega_{+} \supset(\tilde{M} \backslash M)$ and hence $\partial \Omega_{+}$is a MOTS in $M$. As $\partial^{-} M$ was assumed to be an outermost MOTS in $M$, we conclude that the closure of $\Omega_{+}$is $\tilde{M} \backslash M$.

The barriers near $\partial^{+} M$ are so that they imply that the $f_{\tau}$ are uniformly bounded near $\partial^{+} M$. Thus $\Omega_{0}$ contains a neighborhood of $\partial^{+} M$ and $\Omega_{0} \subset M$.

The limit manifold $N$ over $\Omega_{0}$ is a graph satisfying $\mathcal{J}\left[f_{\tau}\right]=0$, and has the desired asymptotics.

We will now discuss a geometric condition to assert that the resulting graph is nonsingular on $M$, i.e., $M=\Omega_{0}$ in Theorem 3.1.

Theorem 3.4. Let $(M, g, K)$ be as in Theorem 3.1 with $\operatorname{tr} K \leq 0$. Then in the assertion of Theorem 3.1 we have that $\Omega_{0}=M$, that is $f$ is defined on $M$ and has no other blow-up than near $\partial^{-} M$. 
Proof. This follows from a simple argument using the maximum principle. Let $f_{\tau}$ be a solution to the regularized problem

$$
\mathcal{H}\left[f_{\tau}\right]-\mathcal{P}\left[f_{\tau}\right]-\tau f_{\tau}=0
$$

in $\tilde{M}$, as in the proof of Theorem 3.1. We claim that $f_{\tau}$ can not have a negative minimum in the region where the data is unmodified. Assume that $x \in M$ is such a minimum. There we have $\mathcal{H}\left[f_{\tau}\right] \geq 0$, and since graph $f$ is horizontal at $x$ we have that

$$
\mathcal{P}\left[f_{\tau}\right]=\operatorname{tr} K \leq 0,
$$

thus the right-hand side of (3.4) is non-negative, whereas $\tau f_{\tau}$ is assumed to be negative, a contradiction.

Since we know that in the limit $\tau \rightarrow 0$, the functions $f_{\tau}$ must blow-up in the modified region which lies in $\Omega_{+}$, we infer a lower bound for $f_{\tau}$ from the above argument. Thus $\Omega_{0}=M$ as claimed.

\section{Asymptotic Behavior}

Here, we discuss a refinement of [SY81, Cor. 2], which says that $N=$ graph $f$ converges uniformly in $C^{2}$ to the cylinder $\partial^{-} M \times \mathbf{R}$ for large values of $f$. A barrier construction allows us to determine the asymptotics of this convergence. Before we present our result, recall the statement of [SY81, Cor. 2]:

Theorem 4.1. Let $N=$ graph $f$ be the manifold constructed in the proof of Theorem 3.1 and let $\Sigma$ be a connected component of $\partial^{-} M$. Let $U$ be a neighborhood of $\Sigma$ with positive distance to $\partial^{-} M \backslash \Sigma$.

Then for all $\varepsilon>0$ there exists $\bar{z}=\bar{z}(\varepsilon)$, depending also on the geometry of $(M, g, K)$, such that $N \cap U \times[\bar{z}, \infty)$ can be written as the graph of a function $u$ over $C_{\bar{z}}:=\Sigma \times[\bar{z}, \infty)$, so that

$$
|u(p, z)|+\left|{ }^{C_{\bar{z}}} \nabla u(p, z)\right|+\left|{ }^{C_{\bar{z}}} \nabla^{2} u(p, z)\right|<\varepsilon
$$

for all $(p, z) \in C_{\bar{z}}$. Here, ${ }^{C_{\bar{z}}} \nabla$ denotes covariant differentiation along $C_{\bar{z}}$.

If $\Sigma$ is strictly stable, we can in fact say more about $u$.

Theorem 4.2. Assume the situation of Theorem 4.1. If in addition $\Sigma$ is strictly stable with principal eigenvalue $\lambda>0$, we have that for all $\delta<\sqrt{\lambda}$ there exists $c=c(\delta)$ depending only on the data $(M, g, K)$ and $\delta$ such that

$$
|u(p, z)|+\left|{ }^{C_{\bar{z}}} \nabla u(p, z)\right|+\left|{ }^{C_{\bar{z}}} \nabla^{2} u(p, z)\right| \leq c \exp (-\delta z) .
$$

Proof. Denote by $\beta>0$ the eigenfunction to the principal eigenvalue $\lambda$ on $\Sigma$ normalized such that $\max _{\Sigma} \beta=1$. We denote by $v$ the normal vector field of $\Sigma$ pointing into $M$. Consider the map

$$
\Psi: \Sigma \times[0, \bar{s}] \rightarrow M:(p, s) \mapsto \exp _{p}^{M}(s \beta \nu) .
$$

Given $\varepsilon>0$ we can choose $\bar{s}>0$ small enough such that the surfaces $\Sigma_{s}=\Psi(\Sigma, s)$ with $s \in[0, \bar{s}]$ form a local foliation near $\Sigma$ with lapse $\beta$ such that

$$
\theta^{+}\left[\Sigma_{s}\right] \geq \lambda(1-\varepsilon) \beta s .
$$


Denote the region swiped out by these $\Sigma_{s}$ by $U_{\bar{s}}$. Note that $\partial U_{\bar{s}}=\Sigma \cup \Sigma_{\bar{s}}$ and $\operatorname{dist}\left(\Sigma_{\bar{s}}, \Sigma\right)>0$. We can assume that $\operatorname{dist}\left(\Sigma_{\bar{s}}, \partial M\right)>0$. On $U_{\bar{s}}$ we consider functions $w$ of the form $w=\phi(s)$. For such functions Jang's operator can be computed as follows:

$$
\mathcal{J}[w]=\frac{\phi^{\prime}}{\beta \sigma} \theta^{+}-\left(1+\frac{\phi^{\prime}}{\beta \sigma}\right) P-\sigma^{-2} K(v, v)+\frac{\phi^{\prime \prime}}{\beta^{2} \sigma^{3}},
$$

where $\sigma^{2}=1+\beta^{-2}\left(\phi^{\prime}\right)^{2}$, and $\phi^{\prime}$ denotes the derivative of $\phi$ with respect to $s$, cf. [AM07]. The quantities $\theta^{+}, K(v, v)$ and $P$ are computed on the respective $\Sigma_{s}$.

Note that with our normalization

$$
\sigma^{-2} \leq \beta^{2}\left|\phi^{\prime}\right|^{-2} \leq\left|\phi^{\prime}\right|^{-2}
$$

and if we assume that $\phi^{\prime} \geq \mu$ for a large $\mu=\mu(\beta)$ we have

$$
\left|1+\frac{\phi^{\prime}}{\beta \sigma}\right| \leq 2\left|\phi^{\prime}\right|^{-2} \text {. }
$$

Furthermore,

$$
\left|\frac{\phi^{\prime \prime}}{\beta^{2} \sigma^{3}}\right|=\frac{\left|\phi^{\prime \prime}\right|}{\beta^{2}\left(1+\beta^{-2} \phi^{\prime 2}\right)^{3 / 2}} \leq \frac{\left|\phi^{\prime \prime}\right|}{\beta^{2}\left(\beta^{-2} \phi^{\prime 2}\right)^{3 / 2}}=\beta \frac{\left|\phi^{\prime \prime}\right|}{\left|\phi^{\prime}\right|^{3}} .
$$

On the other hand, increasing $\mu=\mu(\beta, \varepsilon)$ if necessary, we have

$$
\frac{\left|\phi^{\prime}\right|}{\beta \sigma} \geq 1-\varepsilon,
$$

if $\left|\phi^{\prime}\right| \geq \mu$.

In combination we find that

$$
\mathcal{J}[w] \leq-\lambda(1-\varepsilon) \beta s+\frac{c_{1}}{\left|\phi^{\prime}\right|^{2}}+\beta \frac{\left|\phi^{\prime \prime}\right|}{\left|\phi^{\prime}\right|^{3}},
$$

with $c>0$ depending on $\varepsilon$ and the data $(M, g, K)$, provided $\left|\phi^{\prime}\right| \geq \mu$ and $\phi^{\prime}<0$.

Choosing $\phi(s)=a \log s$ with $a=(1-\varepsilon)^{-1} \lambda^{-1 / 2}$, we calculate that

$$
\phi^{\prime}(s)=\frac{a}{s}, \quad \phi^{\prime \prime}(s)=-\frac{a}{s^{2}},
$$

so that

$$
\frac{1}{\left|\phi^{\prime}\right|^{2}}=\frac{s^{2}}{a^{2}}=(1-\varepsilon)^{2} \lambda s^{2}, \quad \frac{\phi^{\prime \prime}}{\left|\phi^{\prime}\right|^{3}}=\frac{s}{a^{2}}=(1-\varepsilon)^{2} \lambda s .
$$

Thus we can choose $\bar{s}$ so small that $\left|\phi^{\prime}\right| \geq \mu(\beta, \varepsilon)$ and the estimate in (4.2) holds. We can then decrease $\bar{s}$ further, so that $\bar{s} \leq \varepsilon \beta /\left(c_{1}(1-\varepsilon)\right)$. This choice makes the right-hand side of (4.2) non-positive, that is $\mathcal{J}[w] \leq 0$. Hence, we obtain a super-solution $w$ with

$$
\mathcal{J}_{\tau} w \leq 0
$$

at least where $w \geq 0$, that is near $\Sigma$. 
As $w$ blows up near the horizon, and the $f_{\tau}$ are bounded uniformly in $\tau$ on $\Sigma_{\bar{s}}$, we can translate $w$ vertically to $\bar{w}=w+b$ with a suitable $b>0$ so that

$$
\left.f_{\tau}\right|_{\Sigma_{\bar{s}}} \leq\left.\bar{w}\right|_{\Sigma_{\bar{s}}}
$$

for all $\tau>0$. Then the maximum principle implies that $f_{\tau} \leq \bar{w}$ for all $\tau>0$ in $U_{\bar{s}}$ and consequently the function $f$ constructed in Theorem 3.1 also satisfies $f \leq \bar{w}$.

Near $\Sigma$, the graph of $\bar{w}$ can be written as the graph of a function $\bar{v}$ over $\Sigma \times(\bar{z}, \infty)$, where $v$ decays exponentially in $z$. This is due to the fact that by the assumptions on $\beta$, the parameter $s$ is comparable to the distance to $\Sigma$. By the above construction $u \leq v$, where $u$ is the function from Theorem 4.1. Thus we find the claimed estimate for $u$.

Getting the desired estimates for the derivatives of $u$ is then a standard procedure, but as it is a little work to set the stage, we briefly indicate how to proceed.

We choose coordinates of a neighborhood $\Sigma \times \mathbf{R}$ in a slightly different manner as above. Let $\bar{\Psi}: \Sigma \times(-\varepsilon, \varepsilon) \rightarrow M$ be the map

$$
\bar{\Psi}: \Sigma \times(-\varepsilon, \varepsilon) \times \mathbf{R} \rightarrow M \times \mathbf{R}:(x, s, z) \mapsto\left(\exp _{x}(s v), z\right) .
$$

For a function $h$ on $C_{\bar{z}}$ we let $\operatorname{graph}_{\bar{\Psi}} h$ be the set

$$
\operatorname{graph}_{\bar{\Psi}} h=\{\bar{\Psi}(x, h(x), z):(x, z) \in \Sigma \times \mathbf{R}\} .
$$

From Theorem 4.1, it is clear that for large enough $\bar{z}$ the set $N \cap M \times[\bar{z}, \infty)$ can be written as $\operatorname{graph}_{\bar{\Psi}} h$, where $h$ decays exponentially by the above reasoning. We can compute the value of Jang's operator for $h$ as follows:

$$
(\bar{H}-\bar{P})[N]=\mathcal{J} h,
$$

where $\mathcal{J}$ is a quasi-linear elliptic operator of mean curvature type. To be more precise, $\mathcal{J} h$ has the form

$$
\begin{aligned}
\mathcal{J} h= & \partial_{z}^{2} h+\gamma_{h(x, z)}^{i j} \nabla_{i, j}^{2} h-2 \gamma_{h(x, z)}^{i j} \partial_{i}(h) K\left(\partial_{s}, \partial_{j}\right)-\theta^{+}\left[\Sigma_{h(x, z)}\right] \\
& +Q\left(h,{ }^{C_{\bar{z}}} \nabla h,{ }^{C_{\bar{z}}} \nabla^{2} h\right),
\end{aligned}
$$

where $\gamma_{s}$ is the metric on $\Sigma_{s}$ and $Q$ is of the form

$$
Q\left(h,{ }^{C_{\bar{z}}} \nabla h,{ }^{C_{\bar{z}}} \nabla{ }^{2} h\right)=h *{ }^{C_{\bar{z}}} \nabla h+{ }^{C_{\bar{z}}} \nabla h *{ }^{C_{\bar{z}}} \nabla h+{ }^{C_{\bar{z}}} \nabla h *{ }^{C_{\bar{z}}} \nabla h *{ }^{C_{\bar{z}}} \nabla^{2} h,
$$

where $*$ denotes some contraction with a bounded tensor. Furthermore, the vectors $\partial_{i}$, $i=1,2$ denote directions tangential to $\Sigma$ and $\partial_{z}$ the direction along the $\mathbf{R}$-factor in $C_{\bar{z}}$.

By freezing coefficients, we therefore conclude that $h$ satisfies a linear, uniformly elliptic equation of the form

$$
a^{i j} \partial_{i} \partial_{j} h+\left\langle b,{ }^{C_{\bar{z}}} \nabla h\right\rangle-\theta^{+}\left[\Sigma_{h(x, z)}\right]=0 .
$$

By construction we have that $\left|\theta^{+}\left[\Sigma_{s}\right]\right| \leq \kappa s$ for some fixed $\kappa$. Thus $\theta^{+}\left[\Sigma_{h(x, z)}\right]$ decays exponentially in $z$.

Now we are in the position to use standard interior estimates for linear elliptic equations to conclude the decay of higher derivatives of $h$. This decay translates back into the decay of the first and second derivatives of $u$ as the coordinate transformation is smooth and controlled by the geometry of $(M, g, K)$. 
Remark 4.3. If $\Sigma$ is not strictly stable, but has positive $k^{\text {th }}$ variation, we find that the foliation near $\Sigma$ satisfies $\theta^{+}\left[\Sigma_{s}\right] \geq \kappa s^{k}$. Then a function of the form $\phi(s)=a s^{-p}$ with large $a$ and $p=\frac{k-1}{2}$ yields a super-solution. This super-solution can be used to prove that $|u| \leq C z^{2 /(1-k)}$ as above.

We can get even more information about the decay rate. A closer look at Eq. (4.3) yields that the expression for $\mathcal{J} h$ on $C_{\bar{z}}$ can also be written as follows:

$$
\mathcal{J} h=\left(\partial_{z}^{2}-L_{M}\right) h+Q^{\prime}\left(h,{ }^{C_{\bar{z}}} \nabla h,{ }^{C_{\bar{z}}} \nabla^{2} h\right),
$$

since

$$
\begin{aligned}
& \theta_{s}^{+}=s L_{M} 1+\mathcal{O}\left(s^{2}\right), \\
& \gamma_{h(x, z)}^{i j} \nabla_{i, j}^{2} h=\Delta h+Q_{1}\left(h, \nabla h, \nabla^{2} h\right),
\end{aligned}
$$

and

$$
\gamma_{h(x, z)}^{i j} \partial_{i} h K\left(\partial_{s}, \partial_{j}\right)=S(\nabla h)+Q_{2}(h, \nabla h)
$$

where the differential operators $\nabla$ and $\Delta$ are with respect to $\Sigma$. Then note that

$$
L_{M} h=h L_{M} 1-\Delta h+2 S(\nabla h) .
$$

Further investigation of the structure of $Q^{\prime}$ yields that

$$
\left|Q^{\prime}\left(h,{ }^{C_{\bar{z}}} \nabla h,{ }^{C_{\bar{z}}} \nabla^{2} h\right)\right| \leq C\left(|h|^{2}+\left|{ }^{C_{\bar{z}}} \nabla h\right|^{2}+|h|\left|{ }^{C_{\bar{z}}} \nabla^{2} h\right|+\left|{ }^{C_{\bar{z}}} \nabla h\right|^{2}\left|{ }^{C_{\bar{z}}} \nabla^{2} h\right|\right),
$$

So that in view of the differential Harnack estimate $\left|{ }^{C_{\bar{z}}} \nabla h\right| \leq c|h|$ for positive solutions of linear elliptic equations we have that in fact

$$
\left|Q^{\prime}\left(h,{ }^{C_{\bar{z}}} \nabla h,{ }^{C_{\bar{z}}} \nabla^{2} h\right)\right| \leq c|h|\left(|h|+\left|{ }^{C_{\bar{z}}} \nabla h\right|+\left|{ }^{C_{\bar{z}}} \nabla^{2} h\right|\right),
$$

provided $|h| \leq C$. By projecting the equation $\mathcal{J} h=0$ to the one-dimensional eigenspace of $L_{M}$ it is now a somewhat standard ODE argument to show the following result.

Theorem 4.4. Under the assumptions of Theorem 4.2 there are no solutions $h: \Sigma \times$ $[0, \infty) \rightarrow \mathbf{R}$ to the equation

$$
\mathcal{J} h=0
$$

with decay

$$
|h(p, z)|+\left|{ }^{C_{\bar{z}}} \nabla h(p, z)\right|+\left|{ }^{C_{\bar{z}}} \nabla h(p, z)\right| \leq C \exp (-\delta z)
$$

such that $\delta>\sqrt{\lambda}$ and $h>0$. 
Proof. Assume that $h>0$ is such a solution. We derive a contradiction as follows. Let $\lambda$ be the principal eigenvalue and $\phi$ be the corresponding eigenfunction of $L_{M}$ as before. Let $L_{M}^{*}$ be the (formal) adjoint of $L_{M}$ on $L^{2}(\Sigma)$ and denote by $\phi^{*}>0$ its principal eigenfunction, normalized such that $\int_{\Sigma} \phi \phi^{*} \mathrm{~d} \mu=1$. Then the operator

$$
P u=\left(\int_{\Sigma} \phi^{*} u \mathrm{~d} \mu\right) \phi
$$

is a projection onto the eigenspace spanned by $\phi$ and moreover commutes with $L_{M}$. We interpret $h(z)$ as a family of functions on $\Sigma$, that is $h(z)(p)=h(p, z)$ for $p \in \Sigma$. Choose $\alpha(z)$ such that

$$
\operatorname{Ph}(z)=\alpha(z) \phi
$$

and $\beta(z)$ accordingly,

$$
\beta(z) \phi=P\left(Q^{\prime}\left(h(\cdot, z),{ }^{C_{\bar{z}}} \nabla h(\cdot, z),{ }^{C_{\bar{z}}} \nabla^{2} h(\cdot, p)\right)\right) .
$$

Then Eq. (4.4) and the fact that $P$ commutes with $L_{M}$ and $\partial_{z}$ imply

$$
\alpha^{\prime \prime}(z)-\lambda \alpha(z)=\beta(z)
$$

Using $\phi^{*}>0$ and $h>0$ yields $\alpha(z)>0$ and we can furthermore estimate that

$$
\begin{aligned}
|\beta(z)| & \leq c \int_{\Sigma} \phi^{*}|h(p, z)|\left(|h(p, z)|+|\nabla h(p, z)|+\left|\nabla^{2} h(p, z)\right|\right) \mathrm{d} \mu \\
& \leq c \exp (-\delta z) \int_{\Sigma} \phi^{*}|h(p, z)| \mathrm{d} \mu \\
& \leq c \exp (-\delta z) \alpha(z) .
\end{aligned}
$$

Thus, we conclude that on $[\tilde{z}, 0)$ the function $\alpha>0$ satisfies a differential inequality of the form

$$
\alpha^{\prime \prime}(z)-\lambda \alpha \leq \varepsilon \alpha
$$

where $\varepsilon>0$ can be chosen arbitrarily small by choosing $\tilde{z}$ large enough. If $\sqrt{\lambda+\varepsilon}<\delta$ this ODE has no solutions with decay $\exp (-\delta z)$ other than the trivial solution. Thus $\alpha \equiv 0$ and we arrive at the desired contradiction.

Acknowledgement. The author thanks the Mittag-Leffler-Institute, Djursholm, Sweden for hospitality and support during the program Geometry, Analysis, and General Relativity in Fall 2008.

Open Access This article is distributed under the terms of the Creative Commons Attribution Noncommercial License which permits any noncommercial use, distribution, and reproduction in any medium, provided the original author(s) and source are credited. 


\section{References}

[AM07] Andersson, L., Metzger, J.: The area of horizons and the trapped region. Commun. Math. Phys. 290, 941-972 (2009)

[AMS05] Andersson, L., Mars, M., Simon, W.: Local existence of dynamical and trapping horizons. Phys. Rev. Lett. 95, 111102 (2005)

[AMS07] Andersson, L., Mars, M., Simon, W.: Stability of marginally outer trapped surfaces and existence of marginally outer trapped tubes. http://arxiv.org/abs/0704.2889v2[gr-qc], 2007

[Bra01] Bray, H.L.: Proof of the riemannian penrose inequality using the positive mass theorem. J. Diff. Geom. 59(2), 177-267 (2001)

[HI01] Huisken, G., Ilmanen, T.: The inverse mean curvature flow and the riemannian penrose inequality. J. Diff. Geom. 59(3), 353-437 (2001)

[Jan78] Jang, P.S.: On the positivity of energy in general relativity. J. Math. Phys. 19, 1152-1155 (1978)

[Khu09] Khuri, M.: A penrose-like inequality for general initial data sets. Commun. Math. Phys. 290(2), 779-788 (2009)

[MÓM04] Malec, E., Murchadha, N.Ó.: The Jang equation, apparent horizons and the Penrose inequality. Class. Quant. Grav. 21(24), 5777-5787 (2004)

[Sch04] Schoen, R.: Talk Given at the Miami Waves Conference, January 2004

[SY81] Schoen, R., Yau, S.-T.: Proof of the positive mass theorem. II. Commun. Math. Phys. 79(2), 231-260 (1981)

Communicated by P. T. Chruściel 